\title{
Vasospasm is a significant factor in cyclosporine-induced neurotoxicity: Case report
}

\author{
Hilde MH Braakman*1, Jan Lodder1', Alida A Postma², Lambert FR Span³ and Werner H Mess ${ }^{4}$
}

\begin{abstract}
Background: The aetiology of central nervous system lesions observed in cerebral cyclosporine neurotoxicity remains controversial.

Case presentation: We report a 48-year-old woman with a non-severe aplastic anaemia who presented with strokelike episodes while on cyclosporine treatment.

Transcranial Doppler ultrasound revealed severely elevated flow velocities in several cerebral vessels, consistent with vasospasm. Immediately after reducing the cyclosporine dose, the stroke-like episodes disappeared. Only after cyclosporine withdrawal the transcranial Doppler ultrasound abnormalities fully resolved.
\end{abstract}

Conclusions: This case demonstrates a significant role of vasospasm in the pathway of cyclosporine-induced neurotoxicity. Transcranial Doppler ultrasound is an effective tool for the diagnosis and follow-up of cyclosporineinduced vasospasm.

\section{Background}

Cyclosporine A is a potent immunosuppressive agent used in various immunological disorders and is administered to prevent graft rejection of transplantations[1]. Neurotoxicity is the second most common side effect of cyclosporine after nephrotoxicity and appears in up to $60 \%$ of cyclosporine-treated patients[2]. Since predominantly the central nervous system is affected, the clinical symptoms of cyclosporine-mediated neurotoxicity are decreased responsiveness, hallucinations, delusions, seizures, cortical blindness, aphasia, ataxia and stroke-like episodes. Minor symptoms include tremor, agitation, insomnia, anxiety, amnesia, headache, and paraesthesias[1,2]. Central nervous system lesions caused by cyclosporine are located in the border zones between major vascular territories or their main branches[3]. Lesions can occur even when cyclosporine bloodthrough levels are within the therapeutic range[2]. The aetiology of the lesions observed in cerebral cyclosporine neurotoxicity remains controversial.

\footnotetext{
* Correspondence: h.braakman@mumc.nl

${ }^{1}$ Department of Neurology, Maastricht University Medical Centre, Maastricht, the Netherlands

Full list of author information is available at the end of the article
}

\section{Case presentation}

A 48-year-old woman, with a medical history of a nonsevere aplastic anaemia, was re-admitted to the haematology department. She recently had been treated with horse antithymocyte globulin $(15 \mathrm{mg} / \mathrm{kg} /$ day intravenously for 5 days) in combination with high dose cyclosporine and prednisolone, in order to prevent acute serum sickness. Her current oral medication consisted of cyclosporine $350 \mathrm{mg}$ bidaily ( $6 \mathrm{mg} / \mathrm{kg}$ bidaily), prednisone $60 \mathrm{mg}$ once daily, ciprofloxacin $500 \mathrm{mg}$ bidaily and fluconazole $200 \mathrm{mg}$ once daily. At admission, she presented with stroke-like episodes; repetitive episodes of sudden loss of strength in the left leg and numbness on the left side of the face. The motor symptoms resolved within a few minutes to one hour, although a cold feeling in her left leg and left side of her face persisted. She reported no other symptoms. Her vital signs, including blood pressure $(110 / 70 \mathrm{mmHg})$, remained within normal range during these episodes. Neurological examination directly after an episode, revealed no abnormalities besides the disturbed temperature sensations. Furthermore, she was bi-cytopenic (haemoglobin level of 6.1 $\mathrm{mmol} / \mathrm{l}$ with no schistocytes and thrombocyte count of $48 \times 10^{9} / 1$ ) with normal leukocytes (white blood cell count $4.4 \times 10^{9} / 1$ with $50 \%$ granulocytes). Besides the low 


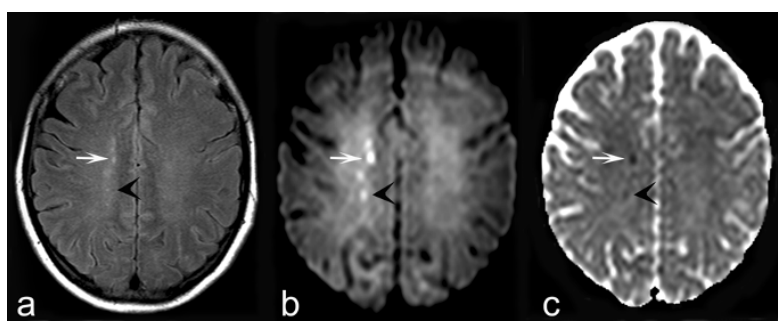

Figure 1 The axial FLAIR image (1A) shows small hyperintense foci in the watershed area in the right hemisphere. Some of these foci show high signal intensity on DWI (1B) and low signal intensity at the ADC-map (1C), consistent with restrictive diffusion (arrows).

thrombocyte count, there were no coagulation disorders. Serum calcium level was $2.07 \mathrm{mmol} / \mathrm{L}$ (normal range 2.10 $\mathrm{mmol} / \mathrm{L}-2.55 \mathrm{mmol} / \mathrm{L}$ ) and serum magnesium level was $0.56 \mathrm{mmol} / \mathrm{L}$ (normal range $0.70 \mathrm{mmol} / \mathrm{L}-1.00 \mathrm{mmol} / \mathrm{L}$ ), for which replenishment was started. Serum cyclosporine concentration was too high $(0.64 \mathrm{mg} / \mathrm{l}$, normal range of $0.10-0.30 \mathrm{mg} / \mathrm{l})$; all other laboratory findings, including renal function and cholesterol level, were normal. Electrocardiogram, 24-hour Holter registration and cardiac ultrasonography revealed no abnormalities. Brain magnetic resonance imaging revealed small hyperintense punctuate foci in the watershed region of the right semioval centre up to the subcortical area between the anterior and middle cerebral arteries (Figure 1A). Some of these small foci showed restricted diffusion using diffusion weighted imaging sequences (Figure 1B and 1C). Lumbar puncture was unremarkable. Extracranial duplex examination showed no abnormalities in the carotid and vertebral arteries. Transcranial Doppler ultrasound revealed segments of severely elevated flow velocities in several cerebral vessels, particularly both middle cerebral arteries (Figure 2A and 2B). These findings were consistent with vasospasm. Based on the toxic cyclosporine concentration in absence of schistocytes (fragmented red blood cells) with no signs of acute serum sickness and thrombotic thrombocytopenic purpura, cyclosporine was reduced with $20 \%$ and fluconazole and prednisone were withdrawn. Immediately after reducing the cyclosporine dose, the stroke-like episodes disappeared. One week after the dose reduction serum cyclosporine concentration had normalized to $0.29 \mathrm{mg} / \mathrm{l}$, but blood flow velocities as measured with transcranial Doppler ultrasound were practically unchanged. Thereupon, cyclosporine was withdrawn. Six weeks after discontinuation of treatment the transcranial Doppler ultrasound abnormalities had fully resolved (Figure 3A and 3B, and Additional file 1: supplemental table 1), the patient remained asymptomatic, and had remission of aplastic anaemia.

\section{Discussion}

The specific distribution of the increased blood flow velocities measured by transcranial Doppler ultrasound, toxic cyclosporine levels and follow-up in our patient clearly demonstrate a significant role of vasospasm in the pathway of cyclosporine-induced neurotoxicity.

Although clinically unlikely, vasculitis or recanalized stenoses of embolic origin may also cause the transcranial Doppler ultrasound changes.

Previous authors have suggested hypertension, direct neurotoxicity, thrombotic microangiopathy, metabolic disturbances, or a combination of these factors as underlying pathophysiology of cyclosporine-induced neurotoxicity[4]. A distinct clinical entity associated with cyclosporine treatment is the "Posterior reversible encephalopathy syndrome" (PRES). PRES is a syndrome clinically characterized by headache, vomiting, confusion, seizures, cortical blindness and other visual abnor-

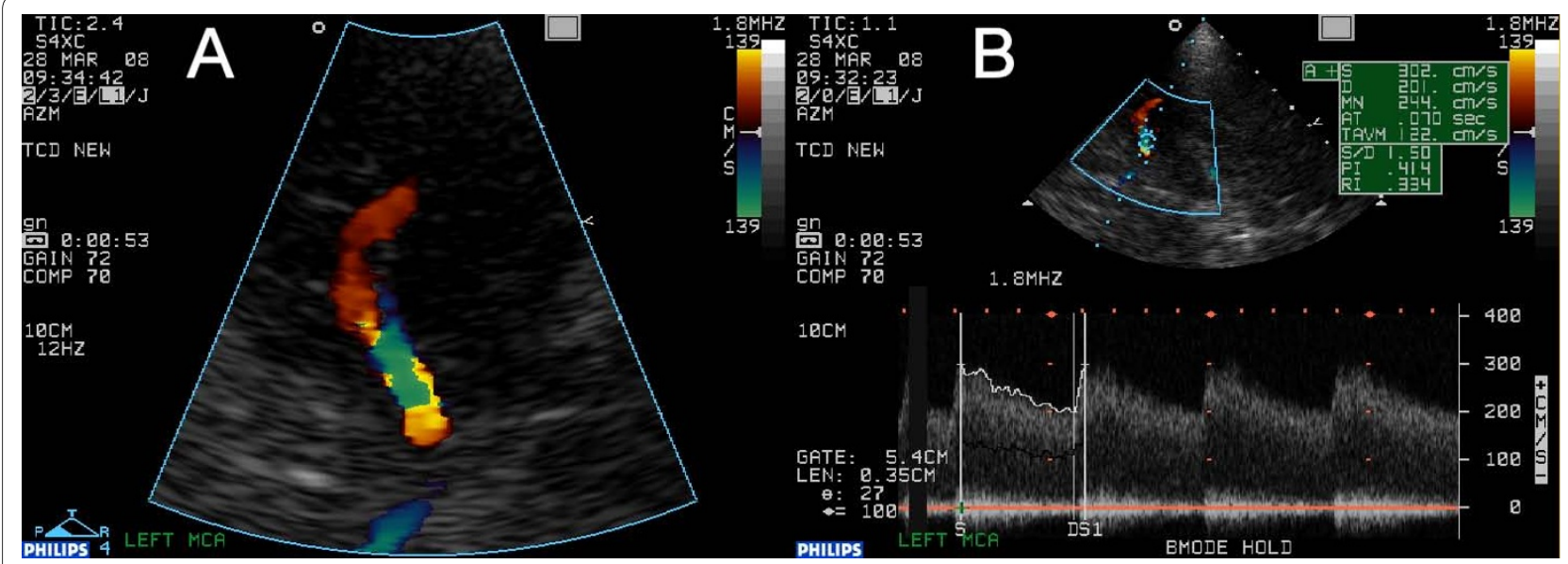

Figure 2 Initial ultrasound findings. On figure 1A, the mainstem and one M2 segment of the left middle cerebral artery are depicted in colour mode using transcranial colour coded duplex sonography. Note the typical aliasing in the most proximal segment despite the rather insensitive setting of the colour scale, indicating a severely stenosed segment. On figure 1B, the Doppler spectogram as measured in that segment is given, indeed showing highly elevated blood flow velocities. 


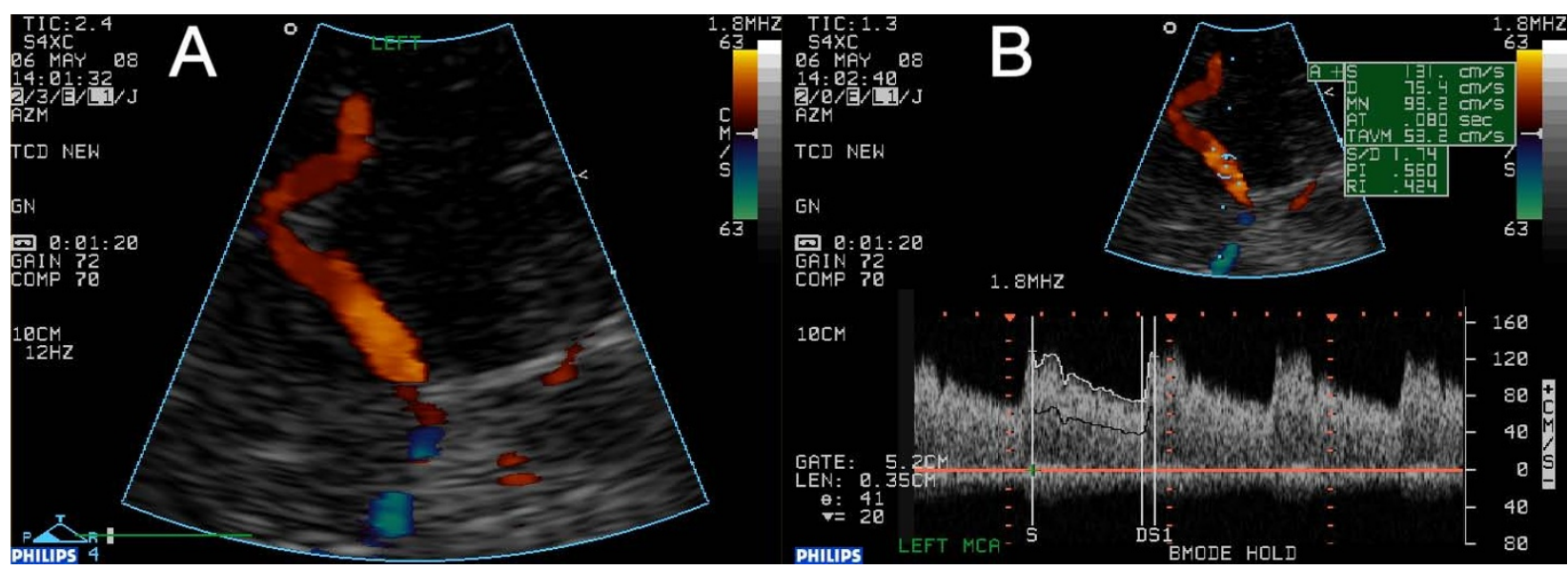

Figure 3 Ultrasound findings six weeks later. The aliasing in the most proximal segment is not present anymore despite a more sensitive setting of the colour scale. Accordingly, the Doppler spectogram shows completely normal blood flow velocities.

malities, and sometimes motor signs, with focal, often symmetric vasogenic edema as its key radiological and pathologic feature [5-8]. The vasogenic edema is thought to result from epithelial dysfunction with vasoconstriction, leukocyte trafficking, or both[9].

In this patient, we believe cyclosporine-induced vasospasm induced this clinical disease. Magnetic resonance imaging revealed focal restricted diffusion, indicative of ischemia, rather than the vasogenic edema of PRES (Figure 1). If cyclosporine therapy is prolonged, the persisting vasospasms may contribute to the development of true PRES, through prolonged hypoxemia leading to endothelial/cellular vascular endothelial growth factor expression, increased vascular permeability and ultimately vasogenic edema. The role of the latter mechanism was highlighted in a recent case report[10].

Although here used in a setting of aplastic anaemia, these findings most likely pertain to all instances where cyclosporine is used, including stem cell and solid organ transplantation. Patients with aplastic anaemia or allogeneic stem cell transplantations receive higher dosages of cyclosporine than solid organ transplanted patients. Therefore, the former category may be at increased risk of cyclosporine neurotoxicity including PRES vasogenic edema.

Cyclosporine-induced neurotoxicity can present in various distinct clinical syndromes; vasospasm as a primary or secondary phenomenon probably plays a key role. Truwit et al were the first who suggested that vasospasm of the intracranial arteries contributed to the mechanism of neurotoxicity. They postulated that endothelial damage plays a primary role, with the release of vasoactive peptides leading to vasospasm as a secondary phenomenon[11]. Later, magnetic resonance angiography was used to assess vasospasm as a central mechanism of cyclosporine neurotoxicity. Interestingly, the lumen nar- rowing was reversible within weeks after therapy cessation $[4,12,13]$. A comparable temporal course was observed in our patient and was also seen by Shbarou et al who described the first two cases in which, next to magnetic resonance angiography, transcranial Doppler ultrasound was used to assess cyclosporine-induced vasospasm[13].

\section{Conclusions}

Central nervous system dysfunction in patients receiving cyclosporine should alert the neurologist to rule out vasospasm of the main arteries of the circle of Willis. Transcranial Doppler ultrasound is a readily available, effective, non-invasive and low-cost tool for the diagnosis and follow-up of cyclosporine-induced vasospasm. Early diagnosis of cyclosporine neurotoxicity is important. The process of vasospasm is reversible in its early stages but in later stages it may become irreversible or even fatal[2].

\section{Consent}

Informed consent was obtained from the patient for publication of this case report and any accompanying images.

\section{Additional material}

\section{Additional file 1 Blood flow velocities measured with transcranial Doppler ultrasound. Blood flow velocities measured with transcranial Doppler ultrasound during cyclosporine treatment (A), One week after cyclosporine dose reduction (B), and six weeks after discontinuation of cyclosporine treatment (C).}

\section{Competing interests}

The authors declare that they have no competing interests.

\section{Authors' contributions}

HMHB collected the data and designed and drafted the manuscript. JL helped to draft the manuscript. AAP analysed the MRI data, drafted the MRI figure legends and helped to draft the manuscript. LFRS participated in the data collection, the design of this article and helped to draft the manuscript. WHM participated in the data collection, the design of this article, helped to draft the 

authors read and approved the final manuscript.

\section{Acknowledgements}

No grants were used for the current study. The authors have no relationships with any entity interested in the subject matter of this manuscript.

\section{Author Details}

'Department of Neurology, Maastricht University Medical Centre, Maastricht, the Netherlands, 2Department of Radiology, Maastricht University Medical Centre, Maastricht, the Netherlands, 3 ${ }^{3}$ epartment of Internal Medicine, Division of Haematology, University Medical Centre Groningen, Groningen, the Netherlands and ${ }^{4}$ Department of Clinical Neurophysiology, Maastricht University Medical Centre, Maastricht, the Netherlands

Received: 5 January 2010 Accepted: 11 May 2010

Published: 11 May 2010

\section{References}

1. Gijtenbeek JMM, Bent MJ van den, Vecht ChJ: Cyclosporine neurotoxicity: a review. J Neurol 1999, 246:339-346.

2. Serkova NJ, Christians U, Benet LZ: Biochemical mechanisms of cyclosporine neurotoxicity. Mol Interv 2004, 4:97-107.

3. Bartynski WS, Grabb BC, Zeigler Z, Lin L, Andrews DF: Watershed imaging features and clinical vascular injury in cyclosporin A neurotoxicity. J Comput Assist Tomogr 1997, 21:872-880.

4. Bartynski WS, Zeigler Z, Spearman MP, Lin L, Shadduck RK, Lister J: Etiology of cortical and white matter lesions in cyclosporin-A and FK506 neurotoxicity. AJNR Am J Neuroradiol 2001, 22:1901-1914.

5. Hinchey J, Chaves C, Appignani B, Breen J, Pao L, Wang A, Pessin MS, Lamy C, Mas JL, Caplan LR: A reversible posterior leukoencephalopathy syndrome. NEng/ J Med 1996, 334:494-500.

6. Greaves P, Oakervee H, Kon SSC, Jones R, Farah N: Posterior reversible encephalopathy syndrome following anti-lymphocyte globulin treatment for severe aplastic anaemia. Br J Haematol 2006, 134:251.

7. Schwartz RB, Bravo SM, Klufas RA, Hsu L, Barnes PD, Robson CD, Antin JH: Cyclosporine neurotoxicity and its relationship to hypertensive encephalopathy: CT and MRI findings in 16 cases. AJR Am J Roentgenol 1995, 165:627-631.

8. Bartynski WS: Posterior reversible encephalopathy syndrome, part 1: fundamental imaging and clinical features. AJNR Am J Neuroradiol 2008, 29:1036-1042.

9. Bartynski WS: Posterior reversible encephalopathy syndrome, part 2: controversies surrounding pathophysiology of vasogenic edema. AJNR Am J Neuroradiol 2008, 29:1043-1049.

10. Horbinski C, Bartynski WS, Carson-Walter E, Hamilton RL, Tan HP, Cheng S: Reversible encephalopathy after cardiac transplantation: histologic evidence of endothelial activation, T-cell specific trafficking, and vascular endothelial growth factor expression. AJNR Am J Neuroradiol 2009, 30:588-590.

11. Truwit $C L$, Denaro $C P$, Lake JR, DeMarco T: $M R$ imaging of reversible cyclosporin A-induced neurotoxicity. AJNR Am J Neuroradiol 1991, 12:651-659.

12. Lin JT, Wang SJ, Fuh JL, Hsiao LT, Lirng JF, Chen PM: Prolonged reversible vasospasm in cyclosporin A-induced encephalopathy. AJNRAm J Neuroradiol 2003, 24:102-104.

13. Shbarou RM, Chao NJ, Morgenlander JC: Cyclosporin A-related cerebral vasculopathy. Bone Marrow Transplantation 2000, 26:801-804.

Pre-publication history

The pre-publication history for this paper can be accessed here: http://www.biomedcentral.com/1471-2377/10/30/prepub

doi: 10.1186/1471-2377-10-30

Cite this article as: Braakman et al., Vasospasm is a significant factor in cyclosporine-induced neurotoxicity: Case report BMC Neurology 2010, 10:30

\section{Submit your next manuscript to BioMed Central and take full advantage of:}

- Convenient online submission

- Thorough peer review

- No space constraints or color figure charges

- Immediate publication on acceptance

- Inclusion in PubMed, CAS, Scopus and Google Scholar

- Research which is freely available for redistribution 